\title{
Deciphering the Synergistic Mechanism of Cortistatin towards Cancer Targets Using Network Pharmacology Approach
}

\author{
Jemmy Christy*, Shiva Shankari, Ilma Majeed, Daniel Alex Anand \\ Department of Bioinformatics, Sathyabama Institute of Science and Technology, Chennai, Tamil Nadu, INDIA.
}

\begin{abstract}
Steroidal alkaloid cortistatin is a promising marine natural compound isolated from marine sponges Corticium simplex. Experimental studies and clinical evidence have shown that cortistatin and its derivatives have a curative effect in patients with autoimmune disorders, HIV infection and several types of cancer. The objective of our study was to examine the potential cancer-related therapeutic objectives of Cortistatin using a network pharmacology method, which is a computational approach, including inverse pharmacophore research, enrichment analysis, molecular docking and dynamics study. Systematic protocol also involves the assessment of ADMET parameters to define the pharmacokinetic profile of cortistatin. Inverse pharmacophore search method was used for computational target fishing and target proteins were ranked based on the graph theory approach. Cancer target proteins, namely HSP90, EGFR, CDK2, MMP13, MAPK13, AR, ESR1, PTPN11 and SRC, were classified as top-ranking proteins according to graph theory parameters, namely MCC, DMNC, MNC, Degree(Local-based methods), EPC, Bottleneck, Eccentricity, Closeness, Radiality, Betweenness, Stress/Global-based methods) and Clustering Coefficient. Enrichment assessment established on Gene Ontology and pathway analysis of these proteins that play a vital role in cancer pathways, FaxO Signalling pathways, Ras Signalling pathways and tyrosine metabolism. Molecular docking and dynamic simulation studies of cortistatin with proposed target proteins were found to be stable and conformers generated after $3 \mathrm{~ns}$ were consistent with stable inter-molecular interactions. The current study summarized here would provide a broad perspective on the therapeutic potential of cortistatin and provide new insights into the future development of cancer therapy strategies.
\end{abstract}

Key words: Cortistatin, Marine natural product, Molecular docking, Network pharmacology, Cancer targets, Pharmacokinetics.

\section{INTRODUCTION}

Marine derived compounds have a variety of chemical structures, functions and therapeutic properties. The marine environment conditions provide the necessary environment to produce secondary metabolites with potentially promising curative bioactivity, which has led to effective clinical findings suggesting the advancement of marineoriginated anticancer drugs. ${ }^{1,2}$ (The National Institutes of Health (NIH) has stated that the total of newly discovered incidents of cancer is projected to rise to 23.6 million per year by 2030. India accounted for the third highest number of cancer cases among women, estimated at 0.7 million in 2017. The very widespread cancers listed are breast cancer, lung and bronchial cancer, prostate cancer, colon cancer, rectal cancer, bladder cancer, non-Hodgkin lymphoma, renal and pelvic cancer, endometrial cancer, leukaemia, pancreatic cancer, skin melanoma thyroid cancer and liver cancer. ${ }^{3}$ The search for new bioactive compounds from marine organisms has resulted in more than 25,700 metabolites to date.
Submission Date: 29-08-2020; Revision Date: 28-07-2021; Accepted Date: 22-10-2021

DOI: 10.5530/ijper.55.4.202 Correspondence: Dr. Jemmy Christy, Department of Bioinformatics, Sathyabama Institute of Science and Technology, Chennai-600119, Tamil Nadu, INDIA.

Phone: +91-944496185, Email-jemmyjacob@gmail. com

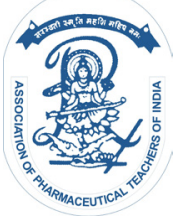

www.ijper.org 
Most of these compounds have pharmacological properties, including anti-tumor, anti-angiogenic, anti-proliferative, cytotoxic, photoprotective, antiinflammatory, anti-oxidant, anti-fouling and antiHIV. ${ }^{4,5}$ Cortistain was one such example, isolated from the Corticium simplex marine sponge for the first time in 2006. This compound is the most potent and selective CDK8 and CDK19 inhibitor. There have also been few preclinical and clinical studies of cortistatin antiHIV potential reported. ${ }^{6}$ In this study, the molecular structure of Cortistain was evaluated according to a variety of parameters, such as rule-of-five, which examined compounds for the definition of physicochemical ranges with a high probability of being an oral drug. ${ }^{7}$ The implementation of computational methodologies for the repurposing of the drug molecules for specified receptor proteins is a key area in the pharmaceutical industry due to its economic benefits and time savings. Computer-assisted, appropriate receptor scanning will be able to enhance drug discovery as well as design activities and thus address current medical needs. Inverse pharmacophore scanning is a promising strategy for the in silico target prediction method. ${ }^{8}$

\section{MATERIALS AND METHODS}

\section{Ligand preparation}

Rational drug design strategy involved two key factors, namely small molecules pharmacophore assessment of the characteristics and affinity to the disease-related biological target. Three dimensional cortistatin structure was recovered from pubchem (SDF format). Clean geometry was used to assess the coordinates of the cutaneous atoms, bond order at the specified location. Molecule was optimized with the CHARMM force field and minimized conformers were subjected to pharmacophore mapping. ${ }^{9}$

\section{Pharmacokinetic properties and drug likeness of cortistatin}

The Swiss ADME web-based server was used to calculate ADME, physicochemistry, drug likeness (Lipinski / Ghose / Veber / Egan / Muegge filters), pharmacokinetics and cortistatin-friendly medicinal chemistry. ${ }^{10}$ In addition, Discovery Studio has been used to predict ADMET Descriptors for example Hepatotoxicity, Blood brain barrier, plasma protein binding and aqueous solubility. The pharmacokinetic properties of Cortistain have been shown to be effective in proposing as a drug compound with high biological activity.

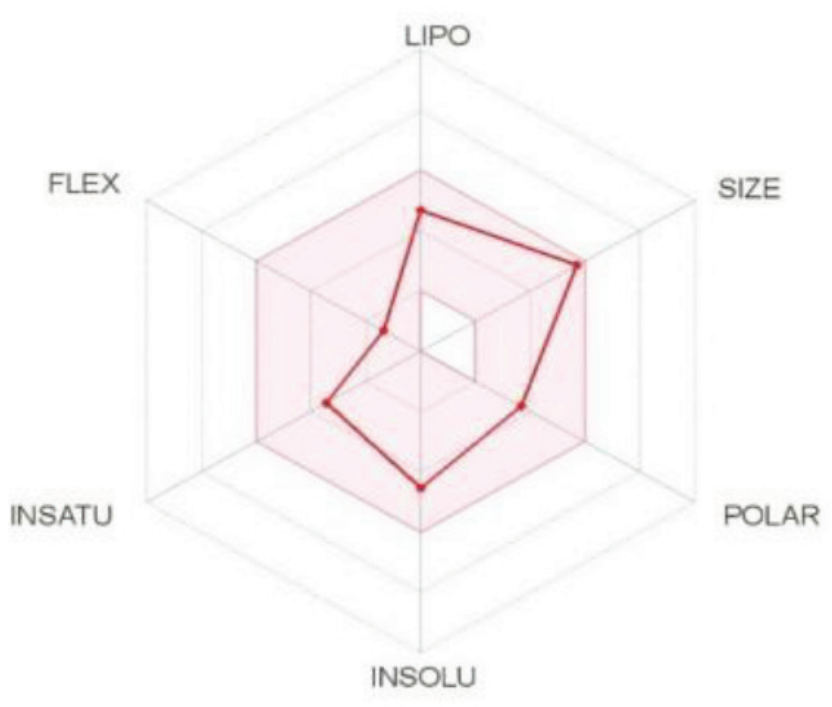

Figure 1: Bioavailability radar; The pink area represents the optimal range of each properties. Plot of polar surface area (PSA) vs LogP for cortistatin, showing $95 \%$ and $99 \%$ of the confidence limit ellipses corresponding to the Blood brain Barrier and intestinal adsorption models. Similarly, the optimal level of hepatotoxicity should be 0 , which means nontoxic; and low penetration; and the PPB should be 0 . These values indicate that Coristatin can be proposed as therapeutic compound.

\section{Inverse pharmacophore-based target retrieval}

The potential targets for cortistatin were predicted by Pharmapper. It is a web server that performs reverse pharmacophore matching using a semi-flexible query compound alignment strategy against the in-house pharmacophore model catalogue, specifically Pharm Target DB glossed information after all the target evidence obtained from Binding DB, Target Bank, Drug Bank and Potential Drug Target Database. ${ }^{11}$ The target proteins with the utmost pairwise fit scores among the pharmacophore models and the query compound remained projected as potential targets.

\section{Network creation and enrichment assessment of cortistatin}

The pharmMapper target selection for Cortistatin resulted in 101 targets from which the top 10 were screened using Cytoscape, an open source software platform for visualization of complex networks and pathways. A highly reliable functional interaction network has been built along the curated pathway. The potential targets obtained are screened using Cytoscape version 3.6.1, an open source bioinformatics software programme for envisioning molecular interactions, pathways and integrating these networks with annotations, 
gene expression profiling data. ${ }^{12}$ First, networks were created using PSICQUIC, which provides programmatic access to molecular interaction databases such as Biogrid $^{13}$ and Reactome ${ }^{14}$ databases.

The PPI networks have been merged into the cytoscape platform and the target list of notes and edges was submitted for enrichment analysis to identify key pathways, ontological terms associated with the proteins listed. To obtain the top 10 proteins, the Cytoscape app called cytohubba ${ }^{15}$ and clueGo ${ }^{16}$ were used. In addition, a pathway analysis was conducted to provide additional support to demonstrate the involvement of proteins in the cancer pathway. These networks were merged manually to obtain imperative nodes / hubs then friable patterns in the interactome network by numerous topological procedures comprising Degree, Edge Percolated Component (EPC), Maximum Neighbourhood Component (MNC), Maximum Neighbourhood Component Density (DMNC), Maximum Clique Closeness, Radiality, Betweenness and Protein Stress Factors in the PPI network.

\section{Protein preparation, ligandability, Druggability screening}

The hub proteins of the PPI network were EGFR, HSP90a, CDK2, MMP13, SRC, AR, ESR1, PTPN11 and MAPK13. The three-dimensional experimental structures were then downloaded using Protein Data Bank (PDB). ${ }^{17}$ Hetero atoms and alternative conformers were then removed to enrich the receptor-ligand interaction study. The cavity algorithm was used to screen binding site residues as well as cavity ligand ability, druggability and pharmacophore characteristics within cavities. ${ }^{18}$

\section{Docking and scoring function}

A total of 9 proteins were docked with the Ligand Fit protocol cortistatin in Discovery Studio, which provides shape-based docking for accurate docking of ligand in protein-active sites. ${ }^{19}$ This approach combines the conformational search of Monte Carlo with the comparison filter of shape for the generation of ligand poses reliable with the active site shape. Cortistatin docked with proposed targets was analysed on the basis of inter-molecular interactions and six score functions, namely LigScores 1 and 2, JAIN, Piecewise Linear Potential (PLP1, PLP2) and Potential of Mean Force (PMF) were used for the calculation of the Dock Score Consensus. ${ }^{20}$

\section{Molecular dynamics and simulation}

Molecular dynamics and simulation findings were used to explore the strength of cortistatin and receptor docking complexes that could result in antagonistic activity within the system. Here we examined the structural integrity of EGFR, HSP90a, CDK2, MMP13, SRC, AR, ESR1, PTPN11 and MAPK13 with Cortistatin in the explicit solvent setting using the Standard Dynamics Cascade (SDC) protocol. CHARMM Force Field ${ }^{9}$ was used to optimize geometry and simulate molecular dynamics. The docked complex geometry was further optimized with the steepest descent and conjugate gradient algorithms. The $300 \mathrm{~K} \mathrm{MD}$ simulations were performed in an implicit water model. Solvent effects were considered with implicit therapy (distance-dependent dielectric constants; dielectric constant $=4$ ). The balancing phase was completed to balance the systems at the target temperature. A molecular dynamic assembly process has been carried out in an appropriate NVT thermodynamic set at a given temperature based on the balanced system from the earlier step. The output of the production stage is stored at 2 ps time interludes. Lastly, the pathways delivered for receptors and their cortistatin complex from the construction run were analysed and evaluated.

\section{RESULTS AND DISCUSSION}

\section{Pharmacokinetic properties and drug likeness profiling of Cortistatin}

According to the Rule of five proposed by Lipinski and Co-workers, cortistatin is orally well absorbed since its molecular weight is $472.62 \mathrm{~g} / \mathrm{mol}$ and consists of two hydrogen bond donors and two hydrogen bond acceptors. The pink area in the Figure 1 represents the optimal range of drug-like properties, i.e. lipophilicity: XLOGP3:2.71, molecular weight:472.62 g / mol,

Polarity:65.82 A22, solubility: moderately soluble, the saturation parameter denotes a fraction of 0.57 carbon during the sp3 hybridization and two rotatable bonds confer flexibility to the compound. Thus, the cortistatin bioavailability score falls within the bioavailability range.

\section{Inverse pharmacophore-based target screening}

Potential cortistatin targets were predicted using the Pharm mapper, the top-listed target proteins were recovered based on the highest ligand-target pairwise fit score. Further refinement was made based on the pharmacophore and fit score and the top 101 cortistatin target proteins were analysed for protein interaction using the Proteomics Standard Initiative Common Query Interface.

\section{Hub protein screening based on centrality parameters}

Screening of cortistatin modulatory effect on pharm mapper projected protein targets protocol initiated 
with the PPI network construction using PSICQUIC platform. PSICQUIC source assist.

The computational read to molecular-interaction data sources by implies of a standard Web Service and query language. Biogrid and the Reactome databases were preferred for the construction of the PPI network. The Biogrid based PPI network was observed with 2771 nodes, 5562 edges and the Reactome network consisting of 2734 nodes and 5919 edges and these two major networks were merged and comprised of 5505 nodes and 11481 edges as shown in Figure 2. Then, through the Cytohubba application in Cytoscape, the merged network, the top 10 nodes (genes) were screened using separate estimation methods, namely MCC, DMNC, MNC, Degree, EPC, Bottleneck, Eccentricity, Closeness, Radiality, Betweenness, Stress and Clustering Coefficient. The intersecting genetic factor derived from these parameters, namely EGFR, HSP90a, CDK2, MMP13, SRC, AR, ESR1, PTPN11 and MAPK13, represented key contender genes with crucial biological regulatory events. These parameters were well-defined as the size of the maximum connected components containing the target nodes and its neighbours. Both modules were highly enriched by oncogenes, tumour suppressors and genes involved in signal transduction as shown in Figure 3. The coloured dots, ranging from deep red to yellow, represent the top 10 genes and their network representation with other genes. The red and yellow rectangles represent the target proteins and the connecting lines represent the correlation and functional associations.

The betweeness of a protein implies the capability of a protein to bring in interaction distant proteins. Whereas the closeness and radiality is inferred as the possibility of a protein to be functionally appropriate for numerous

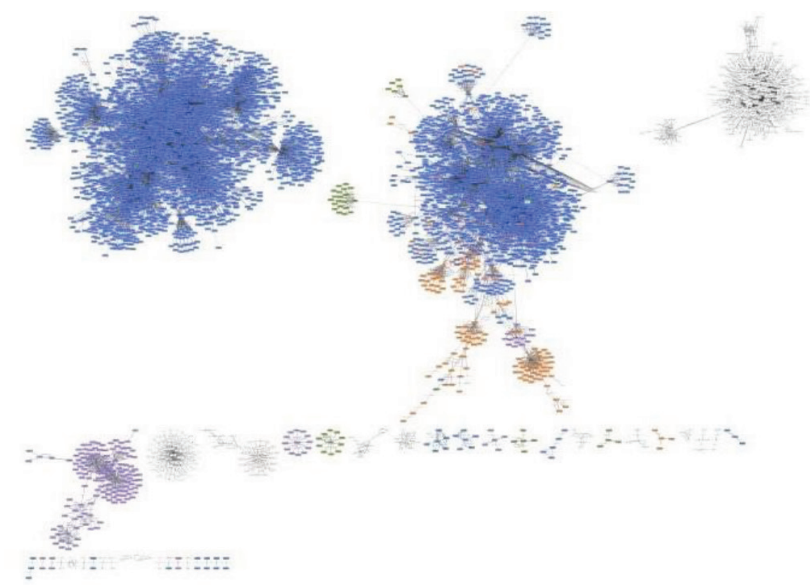

Figure 2: Merged Network of Reactome and Biogrid protein protein interaction network based on pathways and interaction network within the specified system.

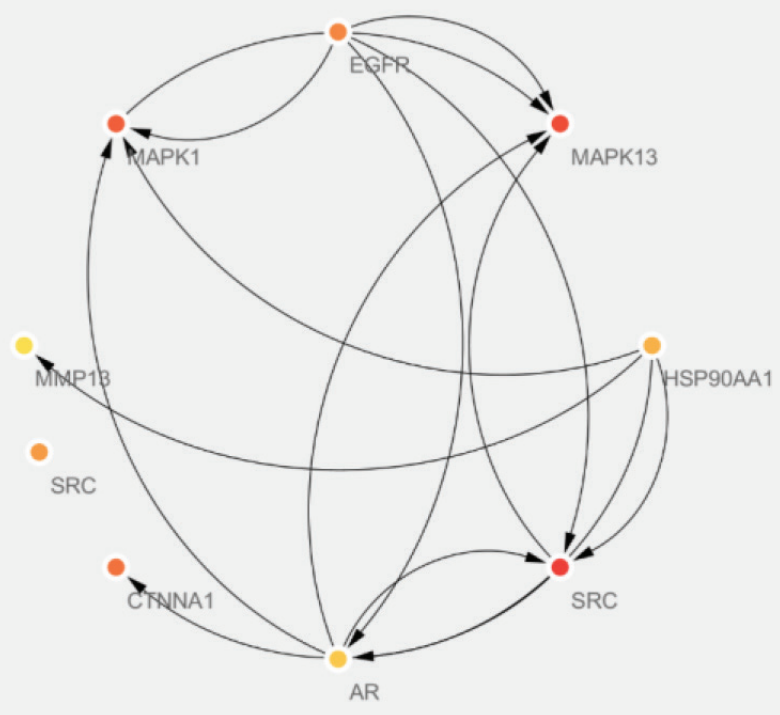

Figure 3: Cytohubba app listed the hub genes based on degree in the protein-protein interaction network. Specified $\mathrm{P}<0.05$ value retrieved the 9 genes.

more proteins and in signalling networks, proteins with very high degree are interacting with several other signalling proteins. The stress indicated the molecule may involve in a cellular process but unable to provide interaction between other proteins. Similarly, the eccentricity of a node in a network explained as the ease of the protein to be functionally attained or influenced by all other proteins. Thus, the detailed protein signalling parameters of the network show the activity and influence of important genes on cancer pathways. Analysis of the problem from the point of view of biological pathways provides the etiology of the disease and GO terms such as biological process, molecular function and cellular component. It indicates the genes associated with the disease or other phenotype and the pathways affected by the genes. Analysis of the hub genes shows that they mainly participated in a 59.12 percent cancer pathway, FoxO signalling pathway, Ras signalling pathway and acute myeloid leukaemia, as shown in Figure 4.

\section{Screening of Hub genes with aberrant expression and clinical significance}

In order to investigate the clinical significance of hub genes, genetic modifications in hub genes were confirmed by engaging cBioportal, an online resource that can examine datasets derived from the TCGA database Figure 5..$^{21,22}$ OncoPrint from cBioportal reported that the predicted hub exhibited genetic alterations, including amplification, significant depletion, mRNA upregulation and variation. Overall Kaplan-Meier Survival estimation of the hub genes is seen in Figure 6. 


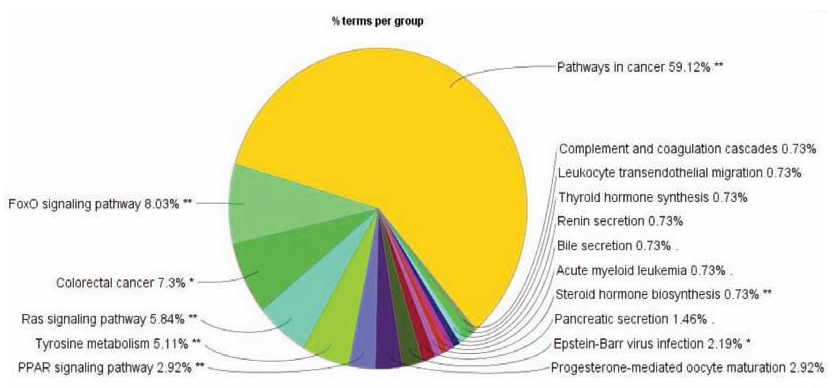

Figure 4: ClueGo app in the cytoscape platform listed the enriched GO network groups related cancer induction.

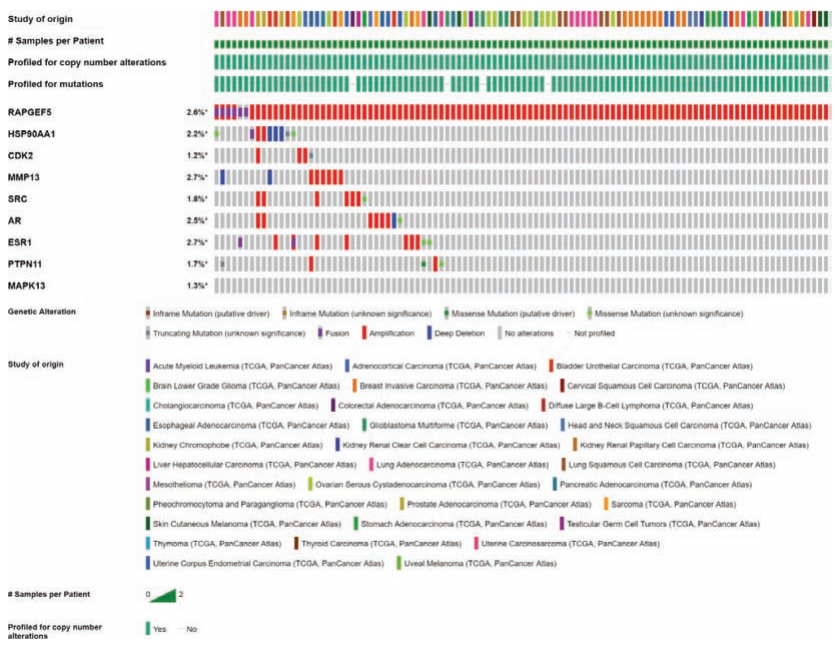

Figure 5: Hub genes aberrant expression and clinical significance using Oncoprint as per TCGA. dataset. Onco Prints are portable ways to represent various genomic modifications, including somatic mutations, alterations in copy numbers, and modifications in mRNA expression across a spectrum of cases.

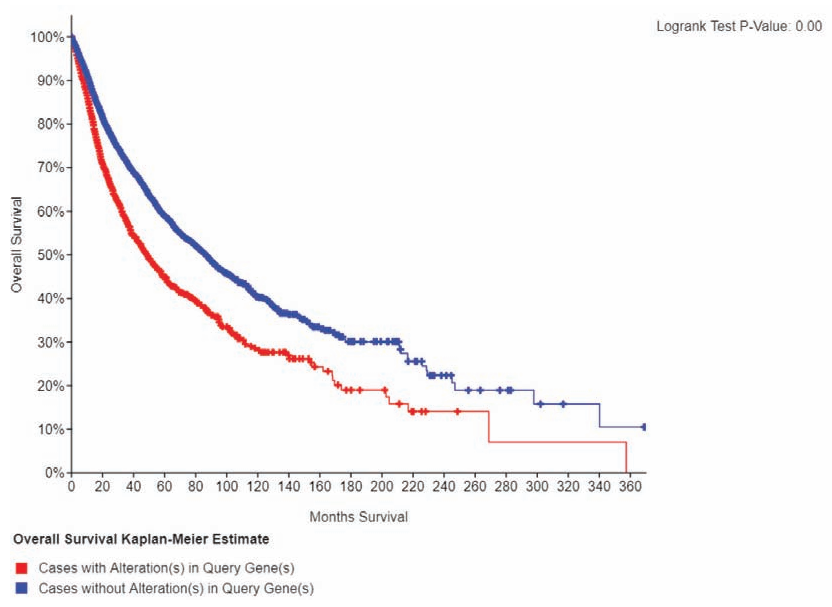

Figure 6: Overall survival Kaplan -Meier estimate for the Hub gene set (HSP90, EGFR, SRC, MMP13, MAPK13, AR, ESR1 and CDK2) differential expression and their evidence-based information on survival time.

\section{Molecular docking, dynamics and simulation analysis}

Molecular docking was systematically used to understand the interactions between the drug receptors. The target protein structures were downloaded from RCSB PDB and for structural enrichment the hetero atoms, ligands and alternative conformers were excluded. Using Discovery Studio 2.1, docking is done by applying the CHARMM force field and the protocol for ligand suit. CHARMM is a common field of all atomic force with a wide range of proteins, nucleic acid and general organic molecules. Then the binding sites are predicted using grid search and ERASER algorithm based on the receptor shape by eliminating all grid points that are not in contact with the receptor, thus identifying receptor cavity sites.

Ligand fit docking occurs as three stages where the docking of ligands or ligands into the binding site of the receptor takes place at stage one, at stage two, in-situ minimization where the ligands can be energy-minimized in the presence of a fixed or partially flexible receptor and various scoring functions are used at the last stage, such as Ligscore1, Ligscore2, PLP1, PLP2, Jain and PMF. These scoring functions assess the structures and binding affinities of the specified cortistatin-receptor complexes. The Jain score is a total of five terms of interaction, such as lipophilic interactions, Polar attractive interactions, Polar repulsive interactions, protein and ligand solving and ligand entropy. LigScore1 and PLP is a fast, easy scoring function for predicting binding affinities between the receptor-ligand. PLP scores are calculated in arbitrary units, with recorded negative PLP scores to make it to them appropriate for use in consensus score computations afterwards. Higher PLP scores demonstrate better binding of the receptorscortistatin complexes. The Candidate ligand poses are evaluated and prioritized according to the Table 1 Dock Score feature. There are two forms of Dock Score. One is centred on a calculation of the force field, the additional on the Piecewise Linear Potential Function (PLP). The energy grid used in our docking analysis is PLP1 and dreiding and the minimisation algorithm is Conjugate Gradient, which uses the prior history of minimization steps and the existing gradient to evaluate the next step. In general, the minimization process is performed to ease the configuration and take off steric overlap that delivers poor interactions.

The docking with the target protein has been studied regarding the following criteria, such as the interaction of amino acids, ligand and protein atoms include bonding with hydrogen and ratings. The dock score obtained for Cortistatin and EGFR complex is 85,465 with 4 carbon, 


\begin{tabular}{|c|c|c|c|c|c|c|c|}
\hline Molecular complex name & 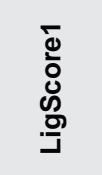 & 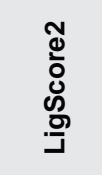 & $\frac{a}{a}$ & $\frac{N}{a}$ & 志 & $\sum_{0}^{L}$ & 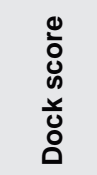 \\
\hline Cortistatin with EGFR & 1.64 & 3.43 & 89.46 & 77.8 & -0.08 & 82.27 & 85.465 \\
\hline Cortistatin with CDK2 & 1.56 & 2.48 & 68.13 & 80.06 & 4.04 & 27.21 & 51.251 \\
\hline Cortistatin with MMP13 & 2.07 & 4.67 & 106.52 & 99.51 & 2.42 & 13.26 & 86.893 \\
\hline Cortistatin with MAPK13 & 3.66 & 4.89 & 76.53 & 79.13 & 0.04 & 58.85 & 76.523 \\
\hline Cortistatin with SRC & -0.82 & -1.54 & 87.87 & 89.72 & 2.22 & 47.24 & 80.594 \\
\hline Cortistatin with HSP90 & -11.97 & -20.34 & 101.45 & 115.35 & 9.34 & 180.88 & 91.065 \\
\hline Cortistatin with ESR1 & 2.45 & 4.18 & 80.55 & 90.1 & 9.44 & 167.73 & 55.241 \\
\hline Cortistatin with AR & -3.26 & -5.79 & 76.76 & 98.85 & 15.84 & 106.53 & 32.541 \\
\hline
\end{tabular}

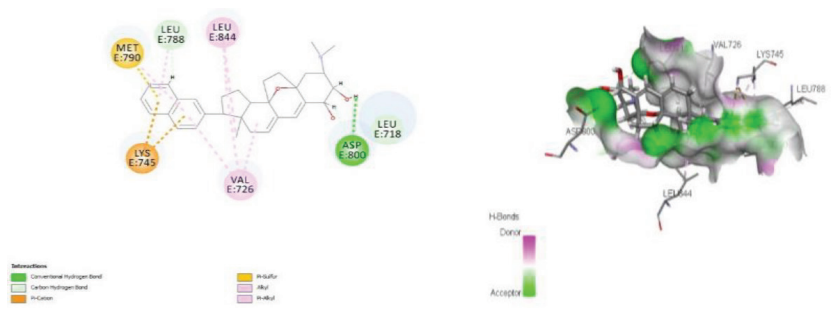

Figure 7: Analysis of interaction between the Cortistatin and EGFR target protein surface with ligand in the interaction defined pocket. He protein and ligand interaction were denoted by dotted lines.

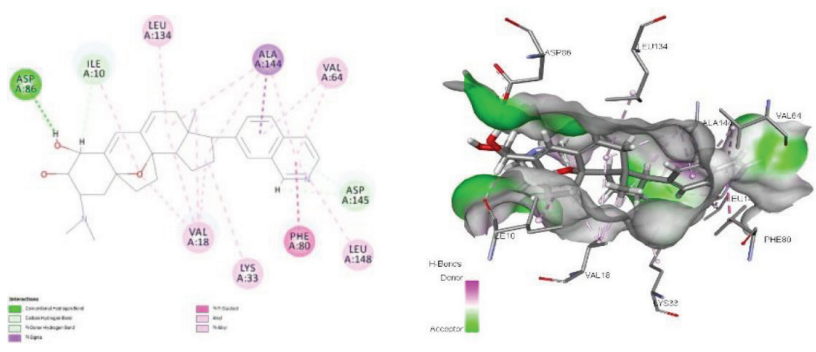

Figure 8: Analysis of interaction between the Cortistatin and CDK2 target protein surface with ligand in the interaction defined pocket. He protein and ligand interaction were denoted by dotted lines.

traditional hydrogen bonds, pi bonds and alkyl bonds present within the EGFR domain regions as shown in (Figure 7), while the dock score for CDK2 is 129,104 and there were 3 hydrogen bond interactions between CDK2 and Cortistatin as shown in (Figure 8).

The promising therapeutic target MMP13, demonstrated a stronger binding affinity with the cortistatin and the dock score of 86,893 was observed. Two hydrogen bonds (Figure 9) have established interactions between
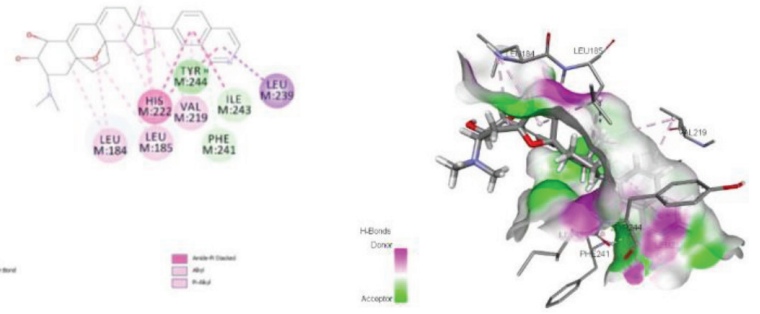

Figure 9: Analysis of interaction between the Cortistatin and MMP13 target protein surface with ligand in the interaction defined pocket. He protein and ligand interaction were denoted by dotted lines.

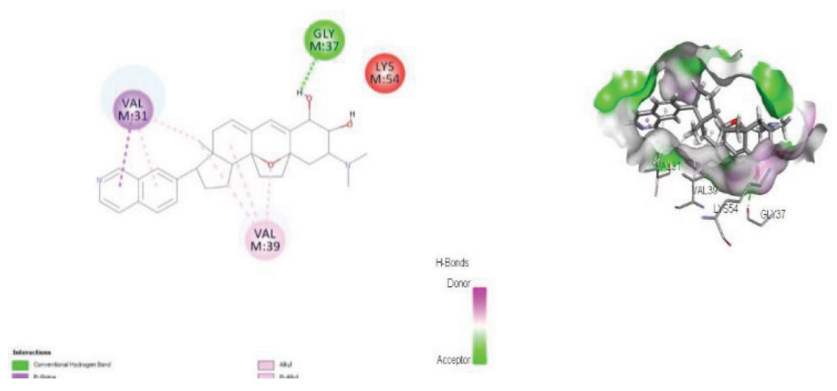

Figure 10: Analysis of interaction between the Cortistatin and MAPK13 target protein surface with ligand in the interaction defined pocket. He protein and ligand interaction were denoted by dotted lines.

MMP13 and cortistatin. Mitogen-activated protein kinase 13 is another promising therapeutic anticancer target showed better binding with the dock score of 76.52 and traditional hydrogen bonds mediate and stabilize the interactions shown in (Figure 10) The interaction-based dock score obtained for cortistatin and SRC is 80.594 with two hydrogen bonds between the molecules lying with the protein domain region (Figure 11). HSP90 mediated four cortistatin hydrogen 


\begin{tabular}{|c|c|c|c|c|}
\hline \multirow[t]{2}{*}{ Ligand } & \multirow[t]{2}{*}{ Amino acid } & \multicolumn{2}{|c|}{ Atom } & \multirow[t]{2}{*}{ Distance } \\
\hline & & Ligand & Receptor & \\
\hline Cortistatin A and EGFR & $\begin{array}{l}\text { ASP } 800 \\
\text { ASP } 800 \\
\text { LEU } 718 \\
\text { LEU } 788\end{array}$ & $\begin{array}{l}\mathrm{H} 61 \\
\mathrm{H} 56 \\
\mathrm{H} 57 \\
\mathrm{H} 69\end{array}$ & $\begin{array}{c}\text { OD2 } \\
\text { OD2 } \\
\text { O } \\
0\end{array}$ & $\begin{array}{c}2.5093 \\
1.75975 \\
2.90109 \\
3.03434\end{array}$ \\
\hline Cortistatin with CDK2 & $\begin{array}{c}\text { ASP } 86 \\
\text { ILE } 10 \\
\text { ASP } 145\end{array}$ & $\begin{array}{l}\mathrm{H} 60 \\
\mathrm{H} 56 \\
\mathrm{H} 69\end{array}$ & $\begin{array}{c}\text { OD1 } \\
\text { O } \\
\text { OD1 }\end{array}$ & $\begin{array}{l}1.82968 \\
2.51815 \\
2.97874\end{array}$ \\
\hline Cortistatin with MMP13 & $\begin{array}{c}\text { PHE } 241 \\
\text { ILE } 243\end{array}$ & $\begin{array}{l}\mathrm{H} 69 \\
\mathrm{H} 69\end{array}$ & $\begin{array}{l}\mathrm{O} \\
\mathrm{O}\end{array}$ & $\begin{array}{l}2.60789 \\
2.91228\end{array}$ \\
\hline Cortistatin A with MAPK13 & GLY 37 & $\mathrm{H} 60$ & $\mathrm{O}$ & 2.04266 \\
\hline Cortisatin A with SRC & $\begin{array}{l}\text { TYR } 340 \\
\text { SER } 342\end{array}$ & $\begin{array}{l}\mathrm{H} 69 \\
\mathrm{H} 69\end{array}$ & $\begin{array}{c}\mathrm{OH} \\
\mathrm{O}\end{array}$ & $\begin{array}{l}2.34851 \\
2.71318\end{array}$ \\
\hline Cortistatin A with HSP90 & $\begin{array}{c}\text { ASP } 93 \\
\text { THR } 184 \\
\text { ILE } 104 \\
\text { ASN } 105\end{array}$ & $\begin{array}{l}\mathrm{H} 61 \\
\mathrm{H} 64 \\
\mathrm{H} 69 \\
\mathrm{H} 69\end{array}$ & $\begin{array}{c}\text { OD2 } \\
\text { OG1 } \\
\text { O } \\
0\end{array}$ & $\begin{array}{c}2.26701 \\
2.1699 \\
2.6821 \\
2.76089\end{array}$ \\
\hline Cortistatin A with ESR1 & $\begin{array}{l}\text { LEU } 346 \\
\text { LEU346 } \\
\text { GLU353 } \\
\text { ALA350 } \\
\text { LEU387 }\end{array}$ & $\begin{array}{r}\mathrm{H} 60 \\
\mathrm{H} 56 \\
\mathrm{H} 61 \\
\mathrm{HA} \\
\mathrm{H} 66\end{array}$ & $\begin{array}{c}\text { O } \\
\text { OE2 } \\
\text { O3 } \\
\text { O } \\
0\end{array}$ & $\begin{array}{c}3.03117 \\
1.97384 \\
2.403 \\
2.21053 \\
2.51799\end{array}$ \\
\hline Cortistatin A with AR & $\begin{array}{l}\text { ASN705 } \\
\text { ASN705 } \\
\text { VAL903 }\end{array}$ & $\begin{array}{l}\mathrm{H} 60 \\
\mathrm{H} 56 \\
\mathrm{HA}\end{array}$ & $\begin{array}{l}\text { OD1 } \\
\text { OD1 } \\
\text { N5 }\end{array}$ & $\begin{array}{c}1.7622 \\
2.87664 \\
2.18962\end{array}$ \\
\hline
\end{tabular}

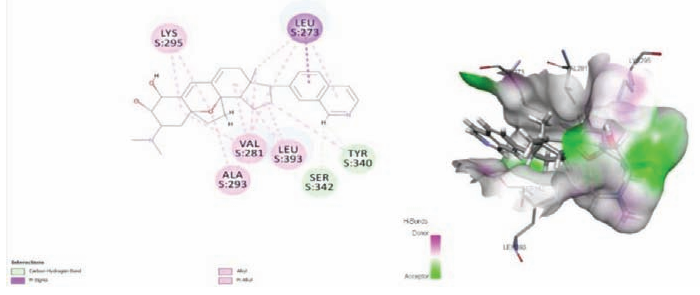

Figure 11: Analysis of interaction between the Cortistatin and SRC target protein surface with ligand in the interaction defined pocket. He protein and ligand interaction were denoted by dotted lines.

bond interactions and the calculated dock score was 91.065 (Figure 12). The binding affinity to cortistatin of the estrogen receptor alpha gene (ESR1) was comparatively small but interactions were within its drug gable cavity residues (Figure 13).

The intensification of the estrogen receptor alpha gene (ESR1) resulted in over-expression of the estrogen receptor protein in different forms of cancer, specifically breast cancer. Our docking studies mediated hydrogen-bonded interactions between cortistatin and ESR1. Similarly, another promising Androgen receptor anticancer target (AR) also showed better binding affinity with cortistatin and binding score
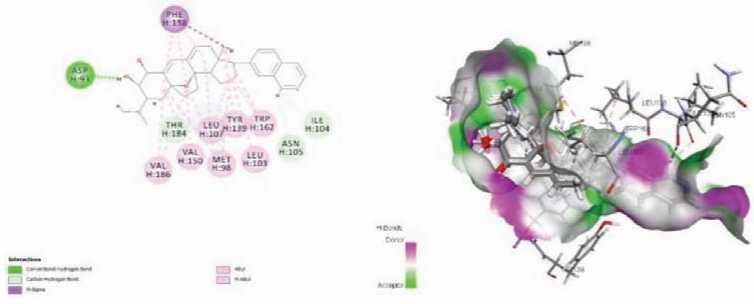

Figure 12: Analysis of interaction between the Cortistatin and HSP90 target protein surface with ligand in the interaction defined pocket. he protein and ligand interaction were denoted by dotted lines.

Figure 14 and Table 2. Molecular dynamics simulation extensively evaluated the actions of cortistatin with its root mean square deviation profiles, binding mode and intermolecular bond counts at the drug gable sites of cancer targets. The result of MD trajectories was 2500 conformations, since we reported conformation at every 2 ps. The presence of intermolecular bonds found in 0 ns was confirmed after $5 \mathrm{~ns}$ MD trajectories at $1 \mathrm{~ns}, 2 \mathrm{~ns}$, $3 \mathrm{~ns}$, $4 \mathrm{~ns}$ and $5 \mathrm{~ns}$ and was not diminished in the case of cortistatin (Figure 15).

In addition, cortistatin complex trajectories were tested for root mean square deviation (RMSD) and root mean 


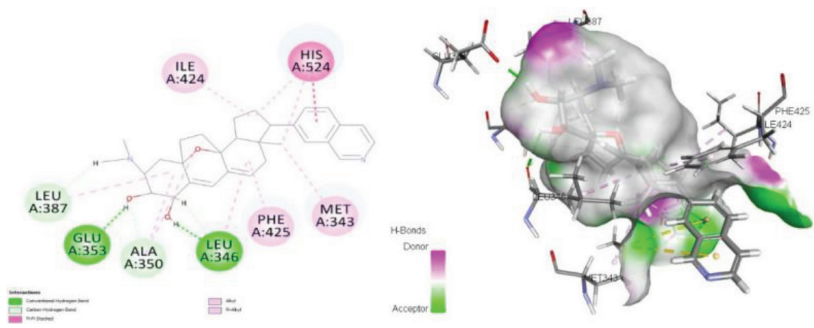

Figure 13: Analysis of interaction between the Cortistatin and ESR1 target protein surface with ligand in the interaction defined pocket. He protein and ligand interaction were denoted by dotted lines.

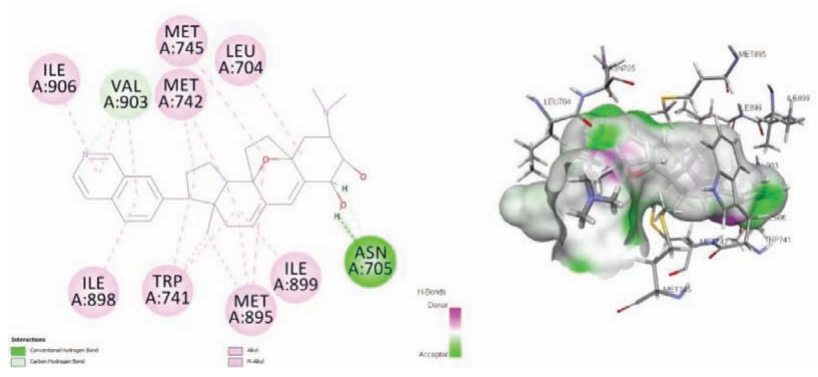

Figure 14: Analysis of interaction between the Cortistatin and AR target protein surface with ligand in the interaction defined pocket. He protein and ligand interaction were denoted by dotted lines.

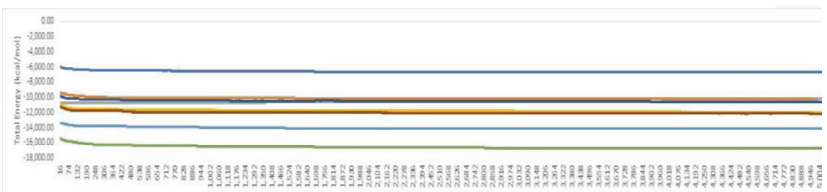

$-102-\infty 4-w-\infty-\infty$

Figure 15: Total energy based simulation observed with the time scale of 5ns, MAPK13 and cortistatin complex energy was comparatively lower than the rest of seven receptor cortistatin complex.

square fluctuation (RMSF) to evaluate the structural level changes. Cortistatin and receptor complex backbone variance $(\mathrm{N}, \mathrm{C} \alpha, \mathrm{C})$ was determined from the RMSD value during MDS. Cortistatin docked complex structures that were observed with variations from 0.7 to 1.9 $\mathrm{A}^{0}$ to equilibrium state (Figure 16). After equilibrium (3ns), the complex system and trend were stabilized and this trend continued up to $5 \mathrm{~ns}$. RMSF values were reported to test the residues with greater flexibility, which in turn affects the rigidity of the docked structure.

Residues with higher RMSF values revealed the residue with more flexibility at 55(EGFR), 73(AR), 139(CDK2),

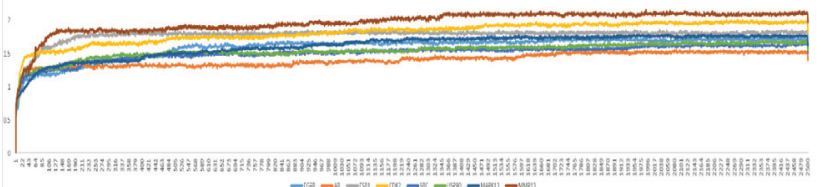

Figure 16: RMSD evalauation of cortistatin and receptor docked complexes is a represention maily for analyzing stability of protein and predicting conformational changes of protein. RMSD.

values depend up on binding interaction and energy between protein and ligand. Our studies revealed that AR, MAPK13 and HSP90 were the optimized protein with lowest RMSD values.

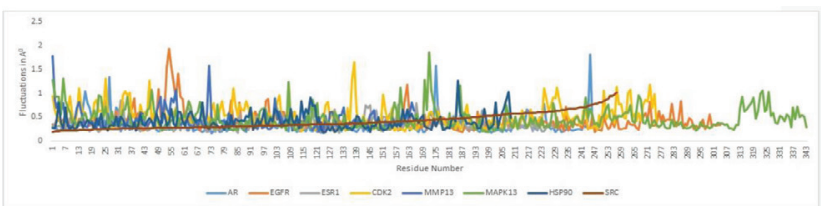

Figure 17: RMSF values in MD trajectory analysis revealed the conformational changes of cortistatin and receptor docked complex.

174(MAPK13) (Figure 17). In general, higher RMSF values meant more flexibility, while smaller RMSF values revealed restricted movement during simulations. ${ }^{23}$

Impoverished pharmacokinetics and toxicity are major causes for expensive late-stage dysfunctions in drug production and it is increasingly accepted that these areas should be identified in the drug discovery phase. Computational methods will improve our competence to calculate and model pharmacokinetic, metabolic and toxicity endpoints, thus transforming the drug development process and accelerating it. Marine derived cortistatin properties meet the criteria and are therefore considered a promising candidate for the expansion of anti-cancer drugs. Using PharmMapper, putative targets are identified for which cytoscape network, gene ontology and pathway analysis have been developed.

The network implied that there are several targets for Cortistatin and further indicated that it has different pharmacological activities. For cortistatin with HSP90, EGFR, SRC, MMP13, MAPK13, AR, ESR1 and CDK2, highly articulated in breast cancer, colorectal cancer, lung cancer and ovarian cancer, molecular docking and subsequent molecular dynamics and simulation studies were performed. Amplification of the ESR1 locus outcomes in overexpression of oncogenic ER protein in the breast and potentially decreases the responsiveness of ESR1 amplified breast tumours to endocrine treatments 
and ultimately contributes to advancement of disease and metastasis. ${ }^{24}$

Selective modulators of estrogen receptors such as cortistatin can function as an antagonist of the ER. Interacting positions LEU 346, LEU346, GLU353, ALA350, LEU387 of ESR1 with cortistatin have been validated with earlier research of pang et al. to screen potent Estrogenic receptor inhibitor design based on in silico research and in vivo method. ${ }^{25}$ Regulation of expression and action of HSP is crucial to blocking their oncogenes is cycle throughout malignancy. In addition to that, HSP protects the tumour cells during cytotoxic therapy and assists the evolutionary process of resistant traits, while storing quasi-stable proteins of the variants.

Such distinct mechanisms perform a crucial role in the advancement and development of tumour cells within the system. ${ }^{26}$ Earlier studies focused on unique pharmacophore characteristics for HSP90 inhibitor design revealed the important amino acid role of HSP90. Key residues namely Asp93, Thr184, ILE 104,ASN 105 mediated interaction with experimental HSP90 inhibitors such as Geldanamycin, Radicicol. ${ }^{27}$ Cortistatin has also shown stable intermolecular interaction with HSP90. Hsp 90 affinity to cortistatin paves the way for more in vitro and vivo studies needed to examine the effect of the antitumor. The rates of expression of MMP-7, MMP-9, MMP-13 and TIMP-1 were elevated in cancer tissue than in neighbouring usual mucous membranes. ${ }^{28}$ MMP13 Expression in the study of the associations among gene expression and clinic opathological factors was observed to associate with liver metastasis. Designbased studies based on structure based MMP13 inhibitor to reveal similar intermolecular interactions such as cortistatin. ${ }^{29}$ Our insilico studies showed the binding affinity of cortistatin inside the MMP13 druggable cavity. MAPK13 over-expression in gynaecological cancers and has functions in the preservation of stem-like cancer cells (CSCs)/cancer-initiating cells (CICs) and tumor-initiating potential. ${ }^{30}$ Thus, intermolecular interaction with cortistatin may render antagonistic activity within MAPK13.

Cycline-dependent kinase-2 (CDK2) performs a crucial function in controlling numerous instances in the eukaryotic cell division process, resulting in irregular cell cycle control resulting in cancer cell hyperproliferation. ${ }^{31}$ So targeting CDK2 with cortistatin could pave the way for the individual affected to have a potential beneficial anticancer effect. The molecule has shown promising interactions with cancer-related proteins in our present research which are responsible for different forms of cancers. Consequently, more refined studies on cortistatin can be undertaken as an anti-cancer target.

\section{CONCLUSION}

Proposing drugs that meet several targets is a drug design challenge. Since they identified several of the active drug targets with minimal structural similarity. While the detection of appropriate targets and drugs performs a crucial role in the design of poly pharmacological modulators. The inverse pharmacophore method has been used in this study to test the suitable therapeutic target for cortistatin. The integrated method was used to screen target proteins based on their druggability, ligandability, hubness in the protein interaction network and aberrant transcriptional profile in cancer tissues. Specific target proteins namely HSP90, EGFR, SRC, MMP13, MAPK13, AR, ESR1 and CDK2 have been identified as appropriate cortistatin targets. Such proteins were associated predominantly with cancer pathways, Faxo signalling pathways, Ras signalling and tyrosine metabolism. Simulation experiments were carried out to test their intermolecular interactions and their stability in further molecular docking. Intermolecular bonding distance, five distinct scoring evaluations to calculate the binding affinity, stability of the docked complexes with a time scale of $5 \mathrm{~ns}$, based on the Dock Score. In docked state MAPK13, HSP90 and CDK2, the potential, kinetic and total energy profile were identified with the promising rank. Stable intermolecular interactions were also observed with other receptors like EGFR, SRC, MMP13, AR, ESR1. To validate the observed intermolecular interaction and associated inhibitor activity of cortistatin, further in vivo and in vitro experiments therefore need to be carried out.

\section{ACKNOWLEDGEMENT}

The authors would like to thank Sathyabama Institute of Science and Technology, Chennai, India for providing the support in carrying out this work.

\section{CONFLICT OF INTEREST}

The authors declare no conflict of interest.

\section{ABBREVIATIONS USED}

RMSD: Root mean square deviation; RMSF: Root mean square fluctuation; GO: Gene Ontology; CHARMM: Chemistry at Harvard Macromolecular Mechanics. 


\section{REFERENCES}

1. Agrawal S, Chaugule S, Indap M. Marine pharmaceuticals: A new wave of antiangiogenic drugs. J Oceanogr. 2018; Res;6:174.

2. Christy $\mathrm{HJ}$, Shankari S. Computational approach to study marine derived cortistatin a molecular mechanism as a Janus kinase 3 inhibitor. Rasayan J Chem. 2020;13(3):1498-505. doi: 10.31788/RJC.2020.1335746.

3. Christy J, Priyadharshini L. Differential expression analysis of JAK/STAT pathway related genes in breast cancer. Meta Gene. 2018;16:122-9. doi: 10.1016/j.mgene.2018.02.008.

4. Ruiz-Torres Verónica, Encinar Jose Antonio, Herranz-López María, PérezSánchez Almudena, Galiano Vicente, Barrajón-Catalán Enrique, Micol Vicente. An updated review on marine anticancer compounds: the use of virtual screening for the discovery of small-molecule cancer drugs. Molecules. 2017;22(7):1037. doi: 10.3390/molecules22071037, PMID 28644406.

5. Mousseau Guillaume, Kessing Cari F, Fromentin Rémi, Trautmann Lydie, Chomont Nicolas, Valente Susana T. The Tat inhibitor didehydro-cortistatin A prevents HIV-1 reactivation from latency. mBio. 2015;6(4):e00465. doi: 10.1128/mBio.00465-15, PMID 26152583.

6. Mousseau Guillaume, Clementz Mark A, Bakeman Wendy N, Nagarsheth Nisha, Cameron Michael, Shi Jun, Baran Phil, Fromentin Rémi, Chomont Nicolas, Valente Susana T. An analog of the natural steroidal alkaloid cortistatin A potently suppresses Tat-dependent HIV transcription. Cell Host Microbe. 2012;12(1):97-108. doi: 10.1016/j.chom.2012.05.016, PMID 22817991.

7. Lipinski CA, Lombardo F, Dominy BW, Feeney PJ. Experimental and computational approaches to estimate solubility and permeability in drug discovery and development settings. Adv Drug Deliv Rev. 2001;46(1-3):3-26. doi: 10.1016/s0169-409x(00)00129-0, PMID 11259830.

8. Byrne R, Schneider G 2019 in silico Target Prediction for Small Molecules. In:.

9. Brooks BR, Bruccoleri RE, Olafson BD, States DJ, Swaminathan S, Karplus M. CHARMM: A program for macromolecular energy, minimization, and dynamics calculations. J Comput Chem. 1983;4(2):187-217. doi: 10.1002/ jcc.540040211.

10. Daina Antoine, Michielin Olivier, Zoete Vincent. SwissADME: a free web tool to evaluate pharmacokinetics, drug-likeness and medicinal chemistry friendliness of small molecules. Sci Rep. 2017;7:42717. doi: 10.1038/ srep42717. PMID 28256516.

11. Liu Xiaofeng, Ouyang Sisheng, Yu Biao, Liu Yabo, Huang Kai, Gong Jiayu, Zheng Siyuan, Li Zhihua, Li Honglin, Jiang Hualiang. PharmMapper server: a web server for potential drug target identification using pharmacophore mapping approach. Nucleic Acids Res. 2010;38(Web Server issue):W609-14. doi: 10.1093/nar/gkq300, PMID 20430828.

12. Shannon Paul, Markiel Andrew, Ozier Owen, Baliga Nitin S, Wang Jonathan T, Ramage Daniel, Amin Nada, Schwikowski Benno, Ideker Trey. Cytoscape: a software environment for integrated models of biomolecular interaction networks. Genome Res. 2003;13(11):2498-504. doi: 10.1101/gr.1239303, PMID 14597658.

13. Oughtred Rose, Stark Chris, Breitkreutz Bobby-Joe, Rust Jennifer, Boucher Lorrie,Chang Christie, Kolas Nadine, O'Donnell Lara, Leung Genie, McAdam Rochelle,Zhang Frederick, Dolma Sonam, Willems Andrew, CoulombeHuntington Jasmin, Chatr-aryamontri Andrew, Dolinski Kara, Tyers Mike. The BioGRID interaction database:2019 update. Nucleic Acids Res. January 08 2019;47(D1):D529-41. doi: 10.1093/nar/gky1079, PMID 30476227.

14. Fabregat A, Jupe S, Matthews L, et al. The reactome pathway KnowledgeBase. Nucleic Acids Res. 2018;46(D1):D649-55.

15. Chin Chia-Hao, Chen Shu-Hwa, Wu Hsin-Hung, Ho Chin-Wen, Ko Ming-Tat Lin Chung-Yen. cytoHubba: identifying hub objects and sub-networks from complex interactome. BMC Syst Biol. 2014;8;Suppl 4 [Suppl:S11]:S11. doi: 10.1186/1752-0509-8-S4-S11, PMID 25521941.

16. Bindea Gabriela, Mlecnik Bernhard, Hackl Hubert, Charoentong Pornpimol, Tosolini Marie, Kirilovsky Amos, Fridman Wolf-Herman, Pagès Franck, Trajanoski Zlatko, Galon Jérôme. ClueGO: a cytoscape plug-in to decipher functionally grouped gene ontology and pathway annotation networks. Bioinformatics. 2009;25(8):1091-3. doi: 10.1093/bioinformatics/btp101, PMID 19237447.
17. Burley Stephen K, Berman Helen M, Bhikadiya Charmi, Bi Chunxiao, Chen L, Di Costanzo Luigi, et al. RCSB Protein Data Bank: biological macromolecular structures enabling research and education in fundamental biology, biomedicine, biotechnology and energy. Nucleic Acids Res. 2019;47(D1):D464-74. doi: 10.1093/nar/gky1004, PMID 30357411.

18. Xu Youjun, Wang Shiwei, Hu Qiwan, Gao Shuaishi, Ma Xiaomin, Zhang Weilin, Shen Yihang, Chen Fangjin, Lai Luhua, Pei Jianfeng. CavityPlus: a web server for protein cavity detection with pharmacophore modelling, allosteric site identification and covalent ligand binding ability prediction. Nucleic Acids Res. July 2 2018;46(W1):W374-9. doi: 10.1093/nar/gky380, PMID 29750256.

19. Venkatachalam CM, Jiang $X$, Oldfield $T$, Waldman M. LigandFit: a novel method for the shape-directed rapid docking of ligands to protein active sites. J Mol Graph Model. 2003;21(4):289-307. doi: 10.1016/s10933263(02)00164-x, PMID 12479928.

20. Jemmy Christy $H$, Swetha. DIFFERENTIAL EXPRESSION ANALYSIS OF KRUPPEL LIKE FACTORS 6 AND ANTAGONISTIC EFFECT STUDY OF CINNAMIC ACID - AN IN SILICO APPROACH. Asian J Pharm Clin Res:107-13. doi: 10.22159/ajpcr.2019.v12i7.33406.

21. Cerami Ethan, Gao Jianjiong, Dogrusoz Ugur, Gross Benjamin E, Sumer Selcuk Onur, Aksoy Bülent Arman, Jacobsen Anders, Byrne Caitlin J, Heuer Michael L, Larsson Erik, Antipin Yevgeniy, Reva Boris, Goldberg Arthur P, Sander Chris, Schultz Nikolaus. The cBio Cancer Genomics Portal: an Open Platform for ExploringMultidimensional Cancer Genomics Data. Cancer Discov. 2012;2(5):401-4. doi: 10.1158/2159-8290.CD-12-0095, PMID 22588877.

22. Gao Jianjiong, Aksoy Bülent Arman, Dogrusoz Ugur, Dresdner Gideon, Gross Benjamin, Sumer SOnur, Sun Yichao, Jacobsen Anders, Sinha Rileen, Larsson Erik, Cerami Ethan, Sander Chris, Schultz Nikolaus. Integrative analysis of complex cancer genomics and clinical profiles using the cBioPortal. Sci Signal. 2013;6(269):pl1. doi: 10.1126/scisignal.2004088, PMID 23550210.

23. Tahir Ul Qamar Muhammad, Maryam Arooma, Muneer Iqra, Xing Feng, Ashfaq Usman Ali, Khan Faheem Ahmed, Anwar Farooq, Geesi Mohammed H, Khalid Rana Rehan, Rauf Sadaf Abdul, Siddiqi Abdul Rauf. Computational screening of medicinal plant phytochemicals to discover potent pan-serotype inhibitors against dengue virus. Sci Rep. 2019;9(1):1433. doi: 10.1038/ s41598-018-38450-1. PMID 30723263.

24. Lei Jonathan T, Gou Xuxu, Seker Sinem, Ellis Matthew J. ESR1 alterations and metastasis in estrogen receptor positive breast cancer. J Cancer Metastasis Treat. 2019;5. doi: 10.20517/2394-4722.2019.12, PMID 31106278.

25. Pang Xiaocong, Fu Weiqi, Wang Jinhua, Kang D, Xu Lvjie, Zhao Ying, Liu Ai-Lin, Du Guan-Hua.Identification of estrogen receptor a antagonists from Natural Products via Invitro and in silico Approaches. Oxid Med Cell Longev. 2018;2018:6040149. doi: 10.1155/2018/6040149.

26. Lang Benjamin J, Guerrero-Giménez Martín Eduardo, Prince Thomas L, Ackerman Andrew, Bonorino Cristina, Calderwood Stuart K. Heat shock proteinS are essential components in transformation and tumor progression: cancer cell intrinsic pathways and beyond. Int J Mol Sci. 2019;20(18):4507. doi: 10.3390/ijms20184507, PMID 31514477.

27. Rampogu Shailima, Parate Shraddha, Parameswaran Saravanan, Park Chanin, Baek Ayoung, Son Minky, Park Yohan, Park Seok Ju, Lee Keun Woo. Natural compounds as potential Hsp90 inhibitors for breast cancerpharmacophore guided molecular modelling studies. Comput Biol Chem. 2019; December;83:107113. doi: 10.1016/j.compbiolchem.2019.107113.

28. Yamada Takanobu, Oshima Takashi, Yoshihara Kazue, Tamura Shuzo, Kanazawa Amane, Inagaki Daisuke, Yamamoto Naoto, Sato Tsutomu, Fujii Shoichi, Numata Kazushi, Kunisaki Chikara, Shiozawa Manabu, Morinaga Soichiro, Akaike Makoto, Rino Yasushi, Tanaka Katsuaki, Masuda Munetaka, Imada Toshio. Overexpression of MMP-13 gene in colorectal cancer with liver metastasis. Anticancer Res. 2010;30(7):2693-9. PMID 20683000.

29. Satish Kumar Kodidasu, Velayutham Ravichandiran, Roy Kuldeep K. A systematic computational analysis of human matrix metalloproteinase 13 (MMP-13) crystal structures and structure-based identification of prospective drug candidates as MMP-13 inhibitors repurposable for osteoarthritis. J Biomol Struct Dyn. 2020;38(10):3074-86. doi: 10.1080/07391102.2019.1651221. PMID 31378153.

30. Yasuda Kazuyo, Hirohashi Yoshihiko, Kuroda Takafumi, Takaya Akari, Kubo Terufumi, Kanaseki Takayuki, Tsukahara Tomohide, Hasegawa Tadashi, 
Saito Tsuyoshi, Sato Noriyuki, Torigoe Toshihiko. MAPK13 is preferentially expressed in gynecological cancer stem cells and has a role in the tumorinitiation. Biochem Biophys Res Commun. 2016;472(4):643-7. doi: 10.1016/j. bbrc.2016.03.004, PMID 26969274
31. Chohan Tahir Ali, Qian Haiyan, Pan Youlu, Chen Jian-Zhong. Cyclin-dependent kinase-2 as a target for cancer therapy: progress in the development of CDK2 inhibitors as anti-cancer agents. Curr Med Chem. 2015;22(2):237-63. doi: 10 $.2174 / 0929867321666141106113633$, PMID 25386824.

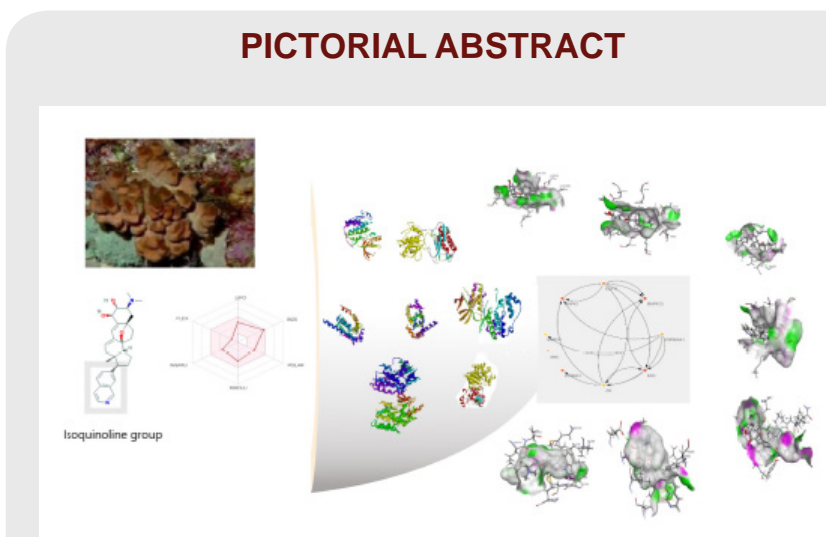

\section{SUMMARY}

Cortistatin, a steroidal alkaloid identified from marine sponges Corticium simplex, is a promising marine natural compound. Cortistatin and its derivatives have been proven to have a curative impact on individuals with immune system related disorders, thus would like to explore their modulatory effect on proteins that are populated in the tumor microenvironment. For computational target fishing, the inverse pharmacophore search method was employed, and target proteins such as HSP90, EGFR, CDK2, MMP13, MAPK13, AR, ESR1, PTPN11, and SRC were prioritised using the graph theory approach. Cortistatin was found to be stable in molecular docking and dynamic modelling investigations with putative target proteins, and conformers formed after 3 ns were consistent with stable intermolecular interactions. The current study, as summarised above, would provide a wide view of cortistatin's therapeutic potential as well as new insights into the future development of cancer therapy.

\section{About Authors}

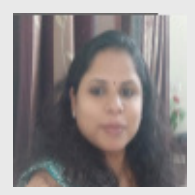

Jemmy Christy: Working as Asst Professor in Department of Bioinformatics, Sathyabama Institute of Science and Technology. Her research interest involves Marine based Insilico drug design in the field of cancer biology.

Shiva Shankari: Post graduate student of Department of Biotechnology, Sathyabama Institute of Science and Technology. Her research interest was computational drug design.

Ilma Majeed: Graduate student of Department of Biotechnology, Sathyabama Institute of Science and Technology. Her research interest was cancer biology.

Daniel Alex Anand: Working as Associate Professor in Department of Bioinformatics, Sathyabama Institute of Science and Technology. His research interest involves systems Pharmacology approach for drug design.

Cite this article: Christy J, Shankari S, Majeed I, Anand DA. Deciphering the Synergistic Mechanism of Cortistatin towards Cancer Targets using Network Pharmacology Approach. Indian J of Pharmaceutical Education and Research. 2021;55(4):1017-27. 